EPJ Web of Conferences 24, 07010 (2012)

DOI: 10.1051/epjconf/20122407010

(C) Owned by the authors, published by EDP Sciences - SIF, 2012

\title{
Optimization of plastic scintillator thicknesses for online beta/gamma detection
}

\author{
K. Pourtangestani* and R. Machrafi \\ University of Ontario Institute of Technology, \\ 2000 Simcoe North, Oshawa, ON, L1H 7K4, Canada
}

\begin{abstract}
For efficient beta detection in a mixed beta gamma field, Monte Carlo simulation models have been built to optimize the thickness of a plastic scintillator, used in a whole body monitor. The simulation has been performed using the MCNP/X code for different thicknesses of plastic scintillator from $150 \mu \mathrm{m}$ to $600 \mu \mathrm{m}$. The relationship between the thickness of the scintillator and the efficiency of the detector has been analyzed. For $150 \mu \mathrm{m}$ thickness, an experimental investigation has been conducted with different beta sources at different positions on the scintillator and the counting efficiency of the unit has been measured. Evaluated data along with experimental ones have been discussed. A thickness of $300 \mu \mathrm{m}$ to $500 \mu \mathrm{m}$ has been found to be the optimum thickness for high efficiency beta detection in the presence of low energy gamma-rays.
\end{abstract}

\section{Introduction}

Detectors have been used not only for investigating the nature of nuclear radiation and their emitters, but they have served also the beneficial application of radiation in medicine, industry, fundamental research, and also the control of any hazards that might arise from ionizing radiation. The

${ }^{*}$ E-mail: kathy.tangesir@gmail.com

This is an Open Access article distributed under the terms of the Creative Commons Attribution License 2.0, which permits unrestricted use, distribution, and reproduction in any medium, provided the original work is properly cited. 
most recent class of detectors developed is one of the solid state detectors. These detectors convert the incident photons directly into electric pulses. Among all detectors, scintillation detectors can be very sensitive because of their higher density, which makes the radiation more likely to be absorbed and detected [1]. Furthermore, plastic scintillators exhibit very short response times and are extensively utilized for experiments where accurate measurements of very short time intervals (ns) must be obtained in spite of prodigiously high count rates. Other common applications are for large area (or volume) multiple-detectors arrays for whole body detectors in nuclear facilities and the detection of charged particles with minimal response to gamma-rays [2]. The CANBERRA Company is a large worldwide company manufacturing radiation instrumentals using plastic scintillators of different kind in a big part of its products. Further investigation was necessary to improve the performance and ISO standard functionality of its products. One of the CANBERRA products using plastic scintillator is the "Argos" full body monitor [6]. The monitor uses several units composed of thin plastic scintillators. However, with the current configuration and layer thicknesses, the system has low detection efficiency for detecting beta and consequently requires long counting times. In this study, a thin plastic scintillator was characterized by simulating its response function by means of detailed MCNP/X simulation as well as by means of calibration measurements for betas and photons at fixed energies. Tests were also conducted at the Canberra facility in order to compare the results with simulation in a mixed radiation beta/gamma field. It is demonstrated that these detectors can be characterized sufficiently enough to serve their function as radiation monitors.

\section{Overview on scintillation detectors}

Organic scintillators are extensively used as radiation detectors. They are found in solid, liquid, and gaseous states. The organic scintillators are aromatic hydrocarbon compounds containing linked or condensed benzene-ring structures. The base material that is generally used for the mixture is an acrylic, a polystyrene, or a polyvinyl-toluene monomer. A convenient scintillator is added to this base and mixed homogeneously. The concentration of this main scintillator is about $1 \%$ by weight of the mixture, which is sufficient to produce a high light yield [3]. Most manufacturers offer different Scintillators based on varying concentration of solvents, so that the user can make the selection according to the system requirements. A plastic 
scintillator produced by this method has several attractive qualities, such as; chemical stability, high degree of optical homogeneity moreover, they can be cut and machined into virtually any shape. Because the material is relatively inexpensive, plastic is often the only practical choice, if large-volume solid scintillators are needed [4]. Their most distinguishing feature is a very rapid decay time on the order of a few nanoseconds or less. The principal scintillation light (or prompt fluorescence) is emitted in transition between higher level and one of the vibration states of the ground electronic state.

\section{Methodology}

The methodology consists of separate steps:

- Characterising the existing unit by measuring its efficiency;

- Start modelling using the MCNP/X simulation tool (Monte Carlo Neutral Particle X) based on the obtained experimental data;

- Further investigation by increasing the thickness of the scintillation layer and perform simulation for different beta sources.

\section{Experiment with existing unit}

At the Canberra site, we have the unit shown below. It consists of one plastic scintillator mounted on a simple plastic for light collection. Thin plastic scintillators are the major sensors used in many detectors and monitors manufactured by the CANBERRA Co. The Argos-TPS monitor 5 PB, recently designed, is using a plastic scintillator of $150 \mu \mathrm{m}$ thickness (fig. 1)

Measurements with the detector unit have been performed for different sources that create a mixed beta-gamma field. The main goal of this testing was to measure the efficiency of the detector with the $150 \mu \mathrm{m}$ scintillation layer and later, to perform the MCNP/X calculations for better optimization. Furthermore, testing included also the increase of the irradiation time and the distance between the source and the detector unit as well. Radiation sources such as ${ }^{14} \mathrm{C},{ }^{60} \mathrm{Co},{ }^{137} \mathrm{Cs}$ and ${ }^{36} \mathrm{Cl}$ have been chosen to match the ones encountered at work places where the monitors are deployed. The characteristics of the radiation sources used in the experiment are listed in table 1.

This experiment has been done just for $150 \mu \mathrm{m}$ thickness for two reasons: the first one is to guide the further $\mathrm{MCNP} / \mathrm{X}$ simulation and the second one, because the unit is a functioning unit at the CANBERRA site. Finally, we 


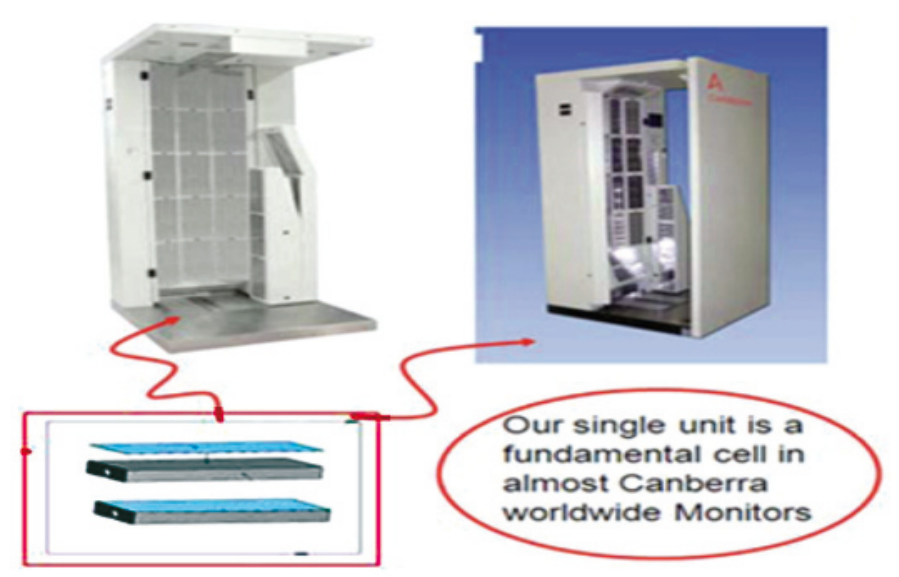

Figure 1: Argos Two-Step (front, back, side, head, hands and feet) whole body surface contamination monitors [6].

Table 1: Characteristics of used sources.

\begin{tabular}{|l|l|l|l|l|l|}
\hline Element & Radiation type & Energy $(\mathrm{MeV})$ & $T_{1 / 2}(\mathrm{y})$ & $A(\mathrm{~Bq})$ & Range $(\mathrm{mm})$ \\
\hline${ }^{14} \mathrm{C}$ & Beta & 0.156 & 5730 & 39627 & 0.28 \\
\hline${ }^{60} \mathrm{Co}$ & Beta-gamma & 0.334 & 5.27 & 7385 & 1.09 \\
\hline${ }^{137} \mathrm{Cs}$ & Beta-gamma & 0.512 & 30.23 & 33600 & 1.78 \\
\hline${ }^{36} \mathrm{Cl}$ & Beta & 0.709 & $3 * 10^{5}$ & 36914 & 2.80 \\
\hline
\end{tabular}

should mention that this test was performed in a low gamma radiation field, which considerably simplified the experiment conditions.

\section{Simulation}

The existing unit at CANBERRA has a thickness of $150 \mu \mathrm{m}$. The goal in this simulation is to determine the optimum thickness of the plastic scintillator to efficiently stop beta radiation and let gamma-rays pass through. The Monte Carlo model was designed and employed to determine the transmission of beta particles and gamma-rays through the scintillation material and to match the physical device as faithfully as possible. Prior to the development of the generic methodology shown in this research, it was necessary to identify the type of model, which achieves the highest rate of photons reaches the photomultiplier tube(PMT) when beta radiation interacts with the plastic scintillator. 


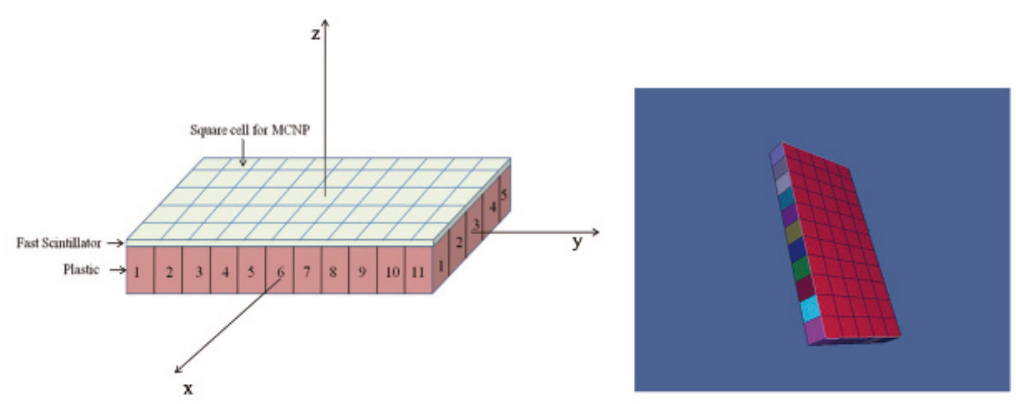

Figure 2: a) 55 cells model to match the size of the PMT, b) Snapshot from the $\mathrm{MCNP} / \mathrm{X}$ Visual Editor.

\section{Different models}

At this stage, by using the MCNP/X code, we built a simple model of the existing detector unit, which matches the exact dimensions of the unit. The model was used to calculate the integrated average flux in the whole plastic box when the unit is irradiated with beta particles as well as with gammarays and to compare the number of photons for both cases. However, this model is very generic and doesn't help to optimize the position of the PMT in the plastic box. Therefore, a second model has been built and it consists of dividing the unit in 55 cells. Each cell has the same dimension as the entrance window of the PMT (fig. 2). Furthermore, this model has been used not only for photon counting in different cells but also for calculating the deposited energy in each cell. The number of photons calculated in the entire plastic box (first model) or in a single cell (second model) didn't help to make a decision regarding the optimization of the thickness of the scintillation layer. The third and last model uses fundamental physics principle for energy deposition. Since the number of photons created in the plastic scintillator depends on the deposited energy, which by itself depends on the incoming particles and the thickness of the scintillation layer, the last model has been built based on calculating the deposited energy in the scintillation layer for different thickness and for beta particles as well as for gamma-rays with different energies. One of the eight tallies of MCNP/X, namely the F8 tally that records the electron and photon energy deposition in each plastic cell was carried and the code was run in different modes: gamma source only, beta radiation only and a mix of beta and gamma radiation. 


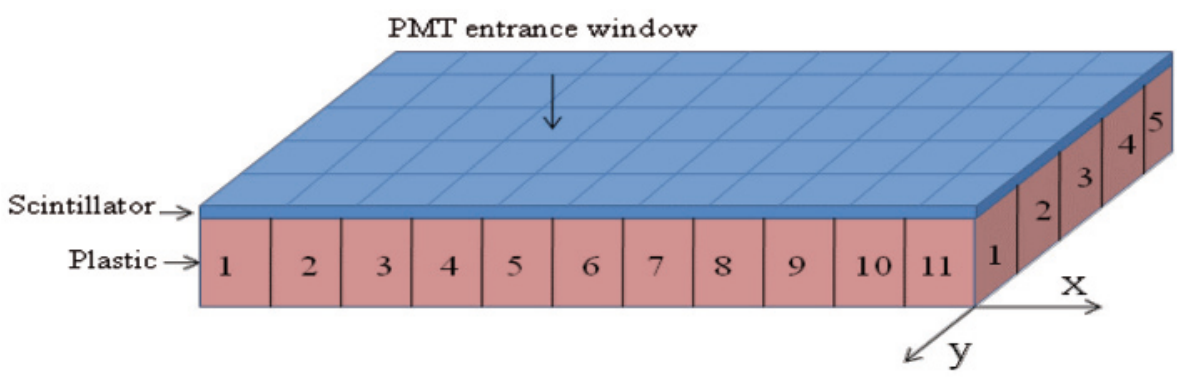

Figure 3: Roadmap of different positions relatively to the PMT.

\section{Result analysis and discussion}

\subsection{Experimental data analysis and discussion}

To analyze the performance of the detector, the experimental data measured with different beta sources placed on the top of the scintillation layer at different positions were converted to 3 dimensional graphs for each experiment; road map of this experiment is shown in fig. 3 .

The variation of the photon-number as function of the position on the $\mathrm{x}$ and $y$ axis are presented in figs. 4 . The further the source is away from the entrance window, the less is the number of counted photons.

\subsection{Simulated data analysis and discussion}

\subsubsection{Beta simulation}

Increasing the thickness of the scintillation layer, as energy goes up, betaparticles deposit more energy in it. For low beta-particles, there is no difference as shown in fig. 5 a by doubling the thickness and in fig. 5 b by increasing the thickness from $150 \mu \mathrm{m}$ to $600 \mu \mathrm{m}$.

As can be seen from fig. 6 below, for low energies we reach saturation in energy deposition once we tripled the thickness. Therefore, there is no sense to increase the thickness anymore. At the same time these two thicknesses should be tested against lowest energy gamma. For this propose we simulated a $60 \mathrm{keV}$ gamma-ray energy.

\subsubsection{Gamma-ray simulation}

The model has been used when the unit was irradiated with gamma sources, as shown in fig. 7 for low gamma-ray energy the amount of deposited energy is high, and by increasing the thickness all energy is deposited. 

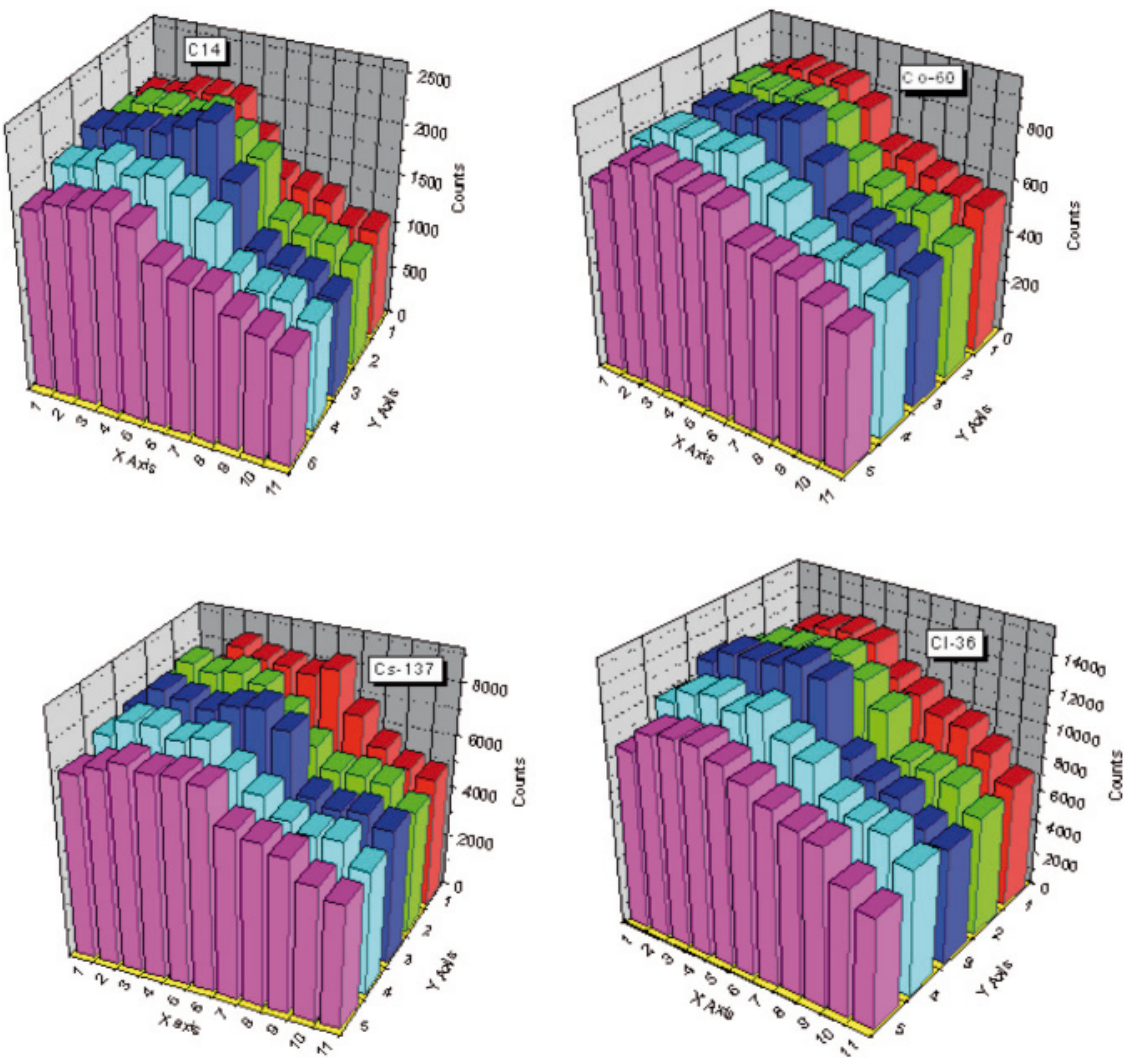

Figure 4: Variation of the photon number as function of the position on the $\mathrm{x}$ - and $\mathrm{y}$-axis for different sources.
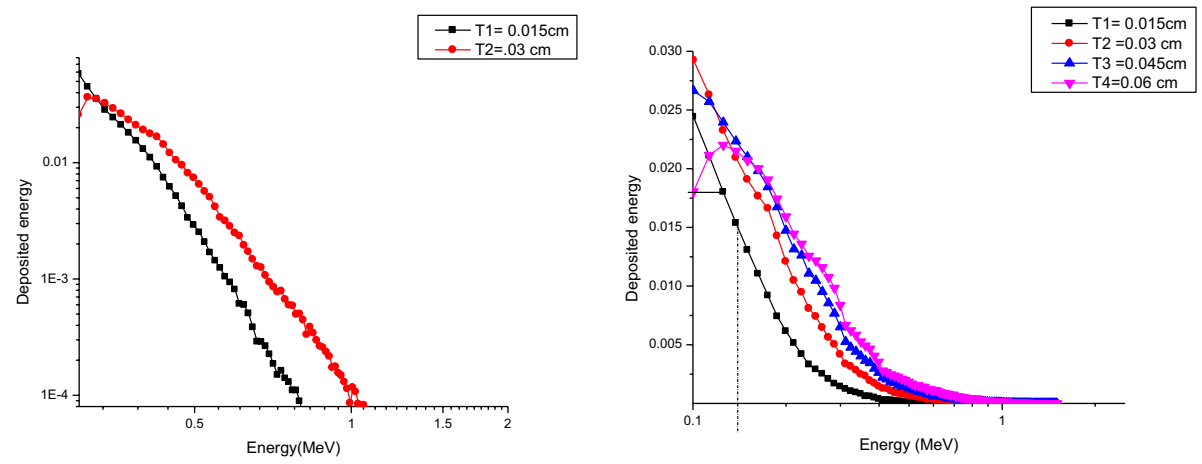

Figure 5: Deposited energy goes up for high beta energy: a) by doubling the thickness of scintillation Layer. b) Increasing thickness from $150 \mu \mathrm{m}$ to $600 \mu \mathrm{m}$. 


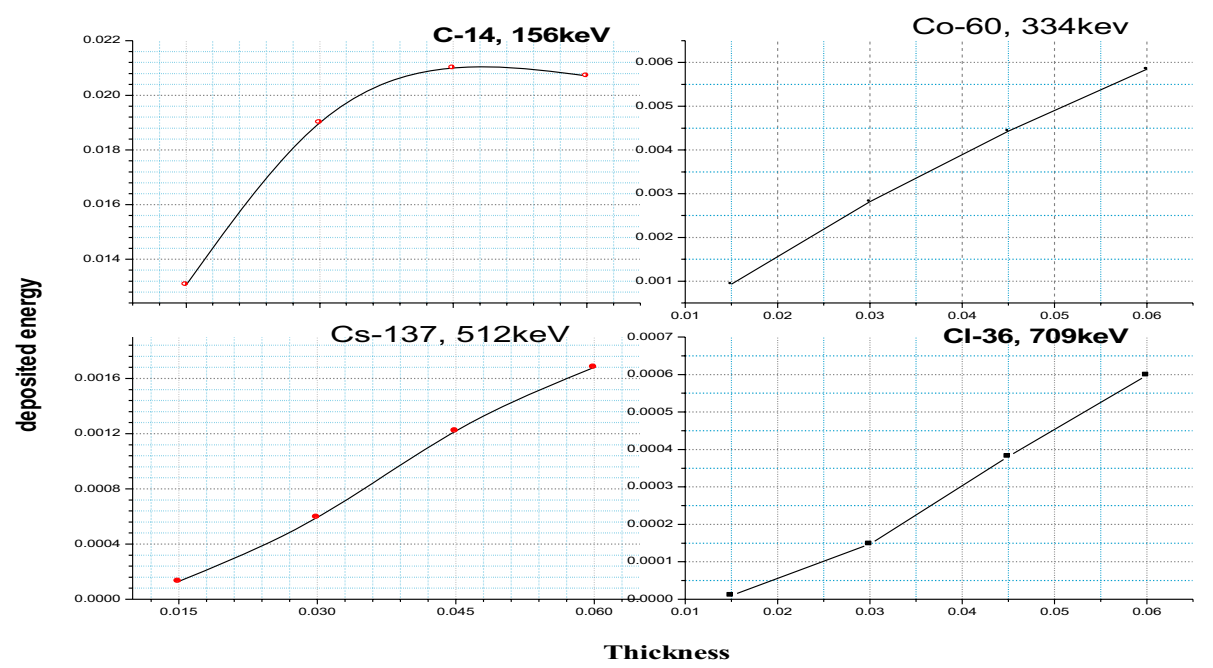

Figure 6: Comparison for different beta energies with different thicknesses.

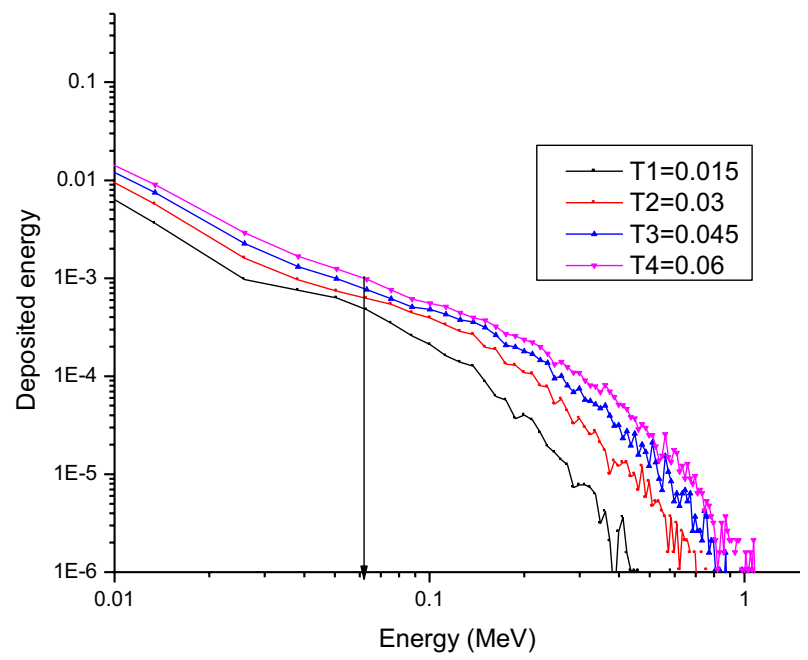

Figure 7: Deposition energy in scintillation as a function of gamma-ray energy and thickness of the scintillation layer. 


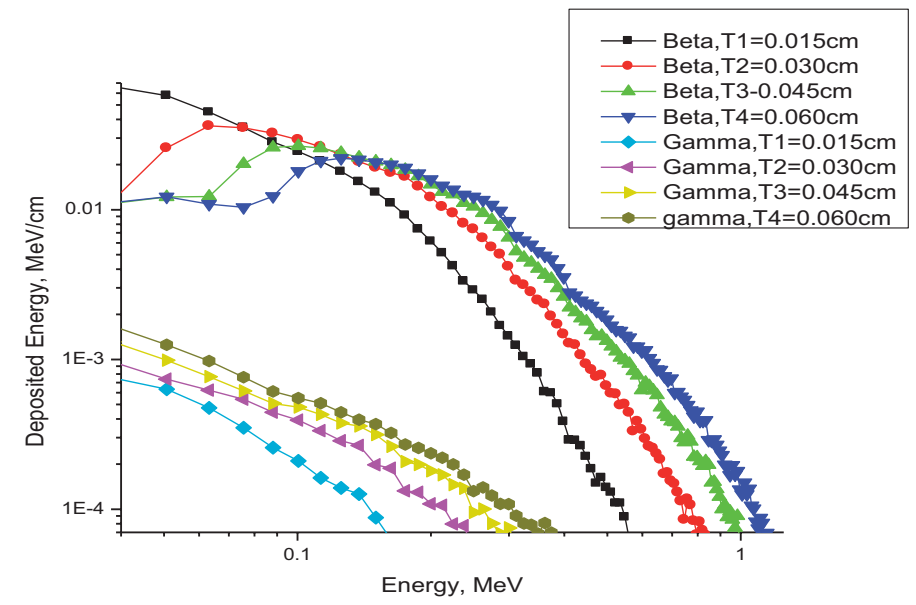

Figure 8: Difference in deposition energy for beta-particle and gamma-ray by increasing thickness of scintillator layer.

Table 2: Beta particles and gamma-rays, deposited energy comparison.

\begin{tabular}{|c|c|c|c|}
\hline $\begin{array}{c}\text { Thickness } \\
\mathrm{cm}\end{array}$ & $\begin{array}{c}\mathrm{D}_{1}=\text { deposition energy } \\
\mathrm{Am}-241,60 \mathrm{keV}\end{array}$ & $\begin{array}{c}\mathrm{D}_{2}=\text { deposition energy } \\
\mathrm{C}-14,156 \mathrm{keV}\end{array}$ & $\mathrm{D}_{1} / \mathrm{D}_{2}$ \\
\hline 0.03 & $6.2215 * 10^{-4}$ & $190 * 10^{-4}$ & $3.2 \%$ \\
\hline 0.045 & $7.6659 * 10^{-4}$ & $210 * 10^{-4}$ & $3.6 \%$ \\
\hline
\end{tabular}

\section{Comparison between beta and gamma deposition energy}

One can see that the energy deposition of beta particles is much higher than for gamma-rays especially when the energy of the gamma-rays is higher than $200 \mathrm{keV}$ (fig. 8).

The comparison between ${ }^{14} \mathrm{C}(156 \mathrm{keV})$ beta particles and low energy gamma-rays ${ }^{241} \mathrm{Am}(60 \mathrm{keV})$ is shown in table 2 ; the ratio of photons coming from gamma-rays is only $3.6 \%$ of that photons coming from beta particles, after interaction with $450 \mu \mathrm{m}$ thickness of scintillation layer.

\section{Deposited beta energy ratio in both layers}

The ratio of the amount of energy deposited in the scintillation layer and in the plastic box is shown in fig. 9 as function of the thickness of the 


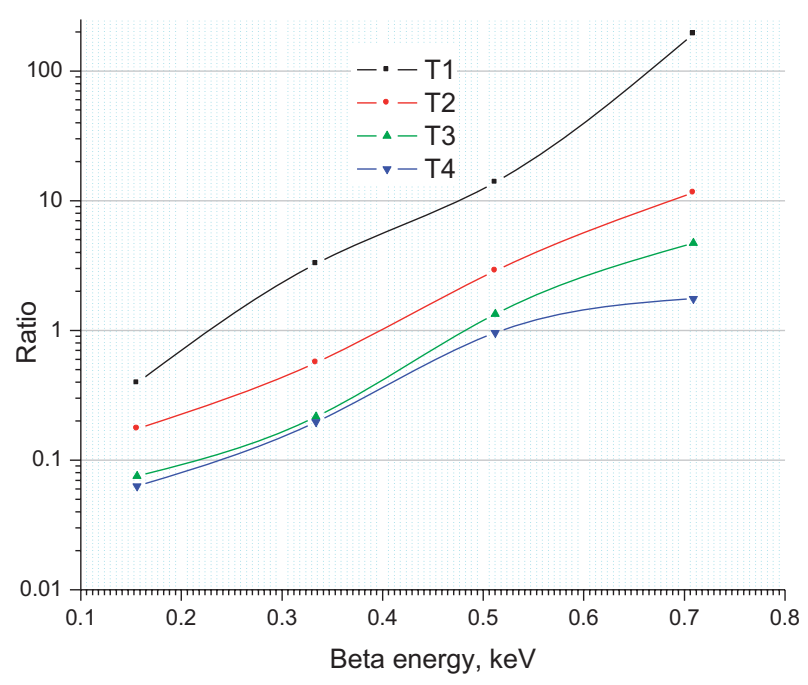

Figure 9: Ratio of deposited energy in the plastic box and in the scintillation layer as function of thickness and beta-particles

scintillation layer.

The ratio of deposited energy is decreasing as function of thickness. The ratio is about $15 \%$ for ${ }^{14} \mathrm{C}$ because of the saturation for the low energy beta. The ratio is dramatically higher for higher beta energies and it is 16, 14 and 110 for ${ }^{60} \mathrm{Co},{ }^{137} \mathrm{Cs}$ and ${ }^{36} \mathrm{Cl}$, respectively. For beta energies of less than $500 \mathrm{keV} 450 \mu \mathrm{m}$ do not make any differences in terms of energy deposition

\section{Conclusion}

Monte Carlo simulation of radiation transport and light collection has been performed to optimize the design parameters for a replacement detector module in order to increase the detector efficiency of beta-particles in plastic scintillators. MCNP/X simulations have been performed to evaluate energies at various locations. A similar approach has been applied to the more complicated case of a mixed beta-gamma radiation field in order to maximize the beta-particle interaction with the scintillation layer and to minimize the gamma-ray interaction. The relationship between the thickness of the scintillation detector and its efficiency has been analyzed. So far we can recommend a value between $0.03 \mathrm{~cm}$ and $0.045 \mathrm{~cm}$ as optimum thickness of the scintillation layer for this detector. By increasing the thickness to $0.03 \mathrm{~cm}$ the deposited energy in scintillation layer for gamma-rays will be 
$3.2 \%$ and for $0.045 \mathrm{~cm}$ it will be $3.6 \%$. As seen for the lowest gamma-rays energy $(\mathrm{Am}-241,60 \mathrm{keV})$ this is the best thickness to let it go through the scintillation layer with the lowest deposited energy besides the fact of having enough thickness for beta particles to deposit most of their energy after interaction with the scintillation layer.

\section{Acknowledgment}

I would like to thank Dr. Rachid Machrafi for supervising me, the CANBERRA Company, which provided this big opportunity to work on a whole body monitor, and UOIT for supporting me during this experiment.

\section{References}

[1] W. R. Leo, Techniques for Nuclear and Particle Physics Experiments, Second Revised Edition, second edition (Springer) 1993, 1994.

[2] Glen F. Knoll, Radiation Detection and Measurement, edition 5 (Wiley), 1989.

[3] James E. Turner; Atoms, Radiation and radiation Protection (Wiley VCh), 2007.

[4] Energy Resolution of Plastic Scintillation Detector for Beta Rays, H. H. Vo, S. Kanamaru, C. Marquet, H. Nakamura, M. Nomachi, F. Piquemal, J. S. Ricol, Y. Sugaya, and K. Yasuda, IEEE trans. nucl. sci., 55 (6) (2008).

[5] Claude Leroy, Pier- Giorgio Rancoita, Principal of Radiation Interaction in Matter and Detection, 2004.

[6] Canberra Company, Measurement Solution for Nuclear Safety and Security, www.canberra.com.

[7] Organic Scintillation Products, Saint-Gobain, 17900 Great Lakes Prkway Hiram, OH 44234 www.detectors.saint-gobain.com, Eljen Technology, Sweetwater TX 79556 USA Website: www.eljentechnology.com.

[8] MCNPXTM User's manual, Denise B. Pelowitz, editor, Version 2.6.0, April 2008. 